A. Yarin, et al.

\title{
CONTROL DE ARMÓNICOS EN TRANSFORMADOR ELÉCTRICO DE POTENCIA DE PLANTA DE MANUFACTURA MEDIANTE FILTRO PASIVO INCORPORADO OPTIMIZADO CON ALGORITMO DE FORRAJE BACTERIAL HARMONIC CONTROL IN POWER PLANT POWER TRANSFORMER BY MEANS OF A BUILT-IN PASSIVE FILTER OPTIMIZED WITH BACTERIAL FORAGING ALGORITHM
}

\author{
Anwar Yarin- Achachagua ${ }^{1^{*} \mathbb{D}}$, Gustavo Salazar- Huamani ${ }^{1} \mathbb{D}$, Javier Chávez- Vivar ${ }^{2} \mathbb{( D}$ \\ ${ }^{1}$ Universidad Nacional Tecnológica de Lima Sur, Facultad de Ingeniería y Gestión, Lima-Perú. \\ ${ }^{2}$ Universidad Nacional de Ingeniería, Facultad de Ingeniería Mecánica, Lima-Perú.
}

Recibido (Received): 04 / 10 / 2020 Aceptado (Accepted): 24/ 04 / 2021

\begin{abstract}
RESUMEN
La presente busca realizar el estudio y caracterización de un filtro pasivo de distorsión armónica aplicado a la protección de una SS.EE de distribución ubicada al interior de una planta de manufactura. El objetivo es diseñar el filtro según los parámetros internos del transformador, reactancia capacitiva e inductiva, y su optimización mediante el método heurístico tipo enjambre de partículas llamado algoritmo de forraje bacterial. El filtro pasivo se sintoniza en el intervalo de frecuencias predominantes de las corrientes armónicas a partir de la inductancia y capacitancia del transformador y una capacitancia externa. El algoritmo de forraje bacterial permitirá diseñar el filtro de manera que contemple el rango de frecuencias de las corrientes armónicas tomando en cuenta las características no lineales de las cargas en aplicación de la estrategia grupal de alimentación de un enjambre de bacterias escherichia coli.
\end{abstract}

La validación experimental se realizó con el equipo CPC 1000microm, que permite obtener las medidas de tangente delta, previa simulación del sistema en el software NEPLAN. Mediante este filtro pasivo rechaza banda se esperan lograr condiciones de trabajo adecuadas y mejoradas atendiendo a las necesidades de proteger tanto el transformador como la maquinaria de planta a la que brinda suministro, especialmente en circunstancias críticas en que los armónicos presentes en la red son altamente agresivos.

Palabras Clave: filtro pasivo de armónicos, inductancia y capacitancia del transformador eléctrico, enjambre de partículas, forraje bacterial escherichia coli.

\section{ABSTRACT}

Through this research we seek to carry out the study and characterization of a passive harmonic distortion filter applied to the protection of a distribution SS.EE located inside a manufacturing plant. The objective is to design the filter according to the internal parameters of the transformer, capacitive and inductive reactance, and its optimization using the particle swarm heuristic method called the bacterial forage algorithm. The passive filter is tuned in the prevailing frequency range of harmonic currents from the inductance and capacitance of the transformer and an external capacitance. The bacterial forage algorithm will be able to design the filter in such a way that it takes into account the frequency range of the harmonic currents, according to non-linear loads in application of the group strategy of feeding a swarm of E. coli bacteria.

The experimental validation was carried out with the CPC 100Omicrom equipment, which allows obtaining the measurements of tan delta, after simulating the system in the NEPLAN software. Through this passive band reject filter, it is hoped to achieve adequate and improved working conditions, meeting the needs of protecting both the transformer and the plant machinery to which it supplies supply, especially in critical circumstances in which the harmonics present in the network are highly aggressive.

Keywords: passive harmonic filter, inductance and capacitance of the electrical transformer, swarm of particles, bacterial forage Escherichia coli.

\footnotetext{
$1^{*}$ Corresponding author:

E-mail: anwaryarin@gmail.com
} 
A. Yarin, et al.

\section{INTRODUCCION}

Los transformadores eléctricos de tensión son máquinas estáticas que consisten en un bobinado primario y secundario, al que la potencia eléctrica suele llegar en alta tensión y sale en baja tensión, quedando definida la relación de tensiones según el número de bobinas en cada extremo. El bobinado secundario va montado normalmente muy próximo al núcleo de hierro, y sobre él va la capa de bobinado de media tensión, la cual presenta el mayor índice de fallas operativas. Esto se debe a que en este bobinado puede generarse un campo eléctrico tan intenso que llega a ionizar el material aislante produciendo descargas eléctricas, que por lo general corresponden con las fallas más graves de un transformador, pudiendo costar la vida de alguna persona. Estas descargas pueden ser francas o parciales [1], siendo esta última materia directa del presente trabajo debido a que la frecuencia de su incidencia se incrementa con la presencia de distorsiones armónicas, pudiendo por tanto prevenirse mediante la implementación del filtro propuesto.

Las fallas de transformadores eléctricos debidas a distorsiones de armónicas están principalmente relacionadas al sobrecalentamiento, que puede llegar a representar directamente el $12 \%$ del total de fallas, y que tiene un efecto deteriorante, reduciendo el tiempo de vida del transformador. Algunas otras fallas que se relacionan indirectamente con fenómenos de sobrecalentamiento son [9]:

- Problemas relacionados con el tanque y fluido
dieléctrico (13\%)
- Cortocircuito de los devanados de un
transformador (19\%)
- Problemas relacionados con el circuito magnético (3\%)

En conjunto, fallas directa o indirectamente relacionadas a las distorsiones armónicas, pueden representar el $50 \%$ de las fallas. Por tanto, estas implican riesgos particularmente altos en el caso de una planta de manufactura debido a los daños que pueden causar a la infraestructura eléctrica y sus equipos, debido a que se traducen en pérdidas económicas importantes por el efecto que puede tener en dichos equipos y el tiempo que se tarde en repararse la falla. En este marco, la sección 2 se orienta a definir el problema y brindar las herramientas previas al diseño del filtro.

Una vez diseñado el filtro en la sección 3, la sección 4 explica la incorporación de un algoritmo de optimización, como el de forraje bacterial, en el diseño del filtro pasivo rechaza banda que se propone permita reducir la probabilidad de falla al reducir el THD. Sin embargo, es importante destacar en contraparte que, el mal diseño o implementación del filtro propuesto, en presencia de una distorsión armónica, puede producir el efecto contrario, amplificándolas y por tanto induciendo un fallo en la SS.EE. De allí la importancia de la simulación en un software de ingeniería como el NEPLAN, que se emplea en la sección 5 para simular el desempeño del filtro de distorsión armónica, previas a las pruebas experimentales controladas que permitan validar las simulaciones.

Finalmente la sección 6 describe brevemente las condiciones experimentales y la sección 7 presenta los resultados comparativamente, en relación a trabajos previos que permitan demostrar la importancia del presente trabajo.

\section{DESCRIPCIÓN Y CARACTERIZACIÓN DEL PROBLEMA \\ 2.1 NOCIONES PREVIAS SOBRE TRANSFORMADORES}

En la actualidad en el contexto industrial no existe un transformador de potencia que presente la capacidad incorporada de protegerse a sí mismo ante la presencia de distorsiones armónicas. En consecuencia, se implementan soluciones parciales al eliminar en lo posible cargas no lineales de la infraestructura eléctrica. Esta solución, sin embargo, limita la variedad de equipos que se pueden instalar, particularmente en el área la manufactura, considerando por ejemplo los siguientes y sus respectivos armónicos típicos [16]:

- Hornos de arco y otros elementos de arco eléctrico, como fluorescentes.

- Controladores de velocidad

- Switches de estado sólido

- Fuentes DC electrónicas y equipos que incorporen rectificadores en cualquier orden de potencia.

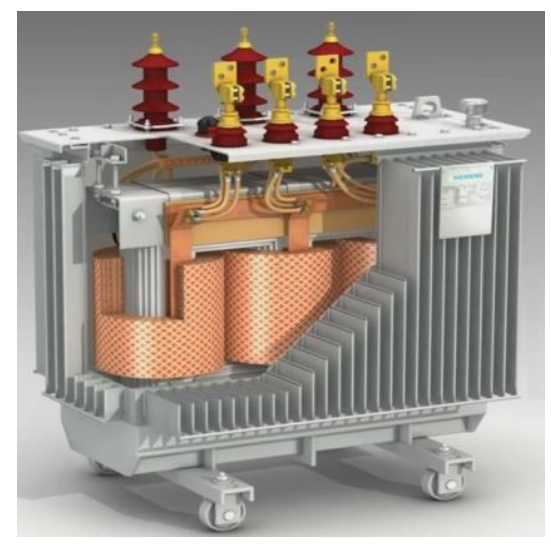

Fig. 1. Disposición general del transformador de potencia.

Para comprender mejor la problemática estudiaremos brevemente los fenómenos que generan las distorsiones armónicas, empezando por repasar la estructura de un transformador. Un transformador trifásico simple está formado por tres columnas, donde para cada uno se disponen devanados monofásicos, 
razón por la que las distorsiones armónicas pueden formarse en cada columna o fase de manera independiente. Así mismo, dado que los devanados de un transformador pueden conectarse en configuraciones diferentes, estas afectan la forma en que las distorsiones armónicas se dispersan en cada devanado. Las configuraciones son en estrella $(Y)$, triangulo (D) y zigzag ( $Z$ ), la última de las cuales presenta limitaciones operativas en cuanto estabilidad térmica relacionada con la generación de armónicos de corrientes (la temperatura de operación suele estar alrededor de $\operatorname{los} 60^{\circ} \mathrm{C}$ ).

Considerando los aspectos constructivos podemos decir que tenemos [3]:

- Núcleo magnético. Encargado de dirigir el flujo magnético producido por el primario.

- Devanados. Constituyen el circuito eléctrico, conducen las corrientes y proporcionan las tensiones adecuadas.

- Cambiadores de tomas. Regulan la tensión en alguno de los devanados para adecuarse a la tensión de la red.

- Bornas. Elementos barrera en la transmisión de la energía de los trafos a la red.

\subsection{DISTORSIONES ARMÓNICAS Y FILTRADO}

Las distorsiones armónicas se producen principalmente como consecuencia de la presencia de cargas no lineales en la arquitectura eléctrica, que para el caso de la industria manufacturera comprenden máquinas o elementos comúnmente encontrados en dispositivos electrónicos o de gran reactancia. Son usualmente de dos tipos según la causa que las genera, normales o característicos y anormales o no usuales. El primer tipo se origina como consecuencia de la presencia de cargas no lineales, mientras que la segunda se genera como consecuencia de otros fenómenos que reproducen condiciones de potencia no lineales, como magnéticos, desbalances de red $y$ periodos transitorios en la generación-transmisión. Estos tipos pueden manifestarse en la red en forma de voltaje o corriente, y en cualquiera de los casos puede modelarse mediante la transformada de Fourier, que permite obtener las frecuencias de las distorsiones armónicas [4].

En el caso de los armónicos de voltaje, uno de sus efectos más dañinos es la generación de picos de tensión elevados que pueden llegar a perforar las bobinas o sobrecalentarlas por periodos prolongados. Esto ocurre particularmente durante la energización y cuando se opera por encima de su tensión normal que los transformadores pueden aumentar considerablemente su contribución armónica [1]. De esta manera, si el transformador funciona dentro del rango nominal, el contenido armónico de la magnetización del transformador en la corriente no es significativa. Por otro lado, los armónicos de corriente tienen el principal efecto de calentamiento y son más fáciles de disipar al implementar una ruta de baja impedancia para los mismos a través de un filtro fijado con la frecuencia del armónico.

Dadas estas características de los armónicos, un parámetro adicional cuando se diseña el filtro pasivo es el incremento repentino de las fuentes de armónicos que puede ser causado por la reconfiguración de cargas o una sobrecarga de red, que finalmente puede generar una falla térmica. Por tanto, para el diseño de un filtro pasivo se debe tener, en la medida de lo posible, un conocimiento preciso de la generación armónica propia de la carga y del sistema de potencia [5].

Con su estructura simple y bajo costo, los filtros pasivos se han usado ampliamente para la mitigación de armónicos, especialmente en la forma de filtros sintonizados y pasa altas. Normalmente se diseñan según el estándar 519 de IEEE, fijando los límites actuales de TDD en el PCC y a la distorsión armónica total de voltaje (THDV) en la red pública. En años más recientes se han implementado filtros activos, sistemas Rectificadores PWM y de filtros híbridos, que incorporan ambos tipos de filtros, los cuales en todos los casos incorporan un hardware y algún algoritmo de control complicado, de los cuales carecen los filtros pasivos y razón por la que han prevalecido en el tiempo con cierto grado de importancia [8].

Bajo este contexto, el diseño del filtro consiste en la sintonización del filtro mediante el fenómeno de resonancia entre $\mathbf{X}_{\mathbf{I}}$, inductor del filtro en el transformador, y $\mathbf{X}_{c}$, la frecuencia fundamental, de manera que la resistencia queda cortocircuitada a esta frecuencia y el filtro opera como un condensador. Para conseguir esta resonancia tanto $\mathbf{X}_{\mathbf{I}}$ y $\mathbf{X}_{\mathbf{c}}$ deben ser iguales [6]. Aun cuando no se generen armónicos $\mathbf{X}_{\mathbf{c}}$ idénticos a los propios del filtro $\mathbf{X}_{\mathbf{l}}$, mientras se desarrolle el régimen estacionario la atenuación aún funciona en los distintos transitorios del sistema [7].

\subsection{INTERCAMBIO DE TOMAS}

Según [19] se tiene que la notación abreviada de la extensión de tomas y de los escalones de regulación indican el rango de variación de la relación de transformación del transformador. Pero esta información no es suficiente para definir los valores asignados de las magnitudes de toma. Esto puede proporcionarse en forma de tabla con la potencia de toma, la tensión de toma y la corriente de toma para cada una, o en forma de texto, indicando la "categoría de regulación de tensión" y las posibles limitaciones a la extensión dentro de la cual las tomas son "tomas de plena potencia". Las categorías extremas de regulación de tensión de toma son: 
- Regulación de la tensión a flujo constante RFC (figura 2.a): La tensión de toma $U_{B}$ en el arrollamiento sin toma es constante, mientras que la tensión con toma $\mathbf{U}_{\mathbf{A}}$ son proporcionales a los factores de toma, observándose fenómeno inverso en la corriente $\mathrm{I}_{\mathrm{A}}$. Se aplica para tomas con factores de toma inferiores al factor de toma de tensión máxima.
- Regulación de la tensión a flujo a variable RFV (figura 2.b): La tensión de toma $\mathbf{U}_{\mathbf{A}}$ en el arrollamiento con toma es constante. Las tensiones de toma en cualquier arrollamiento sin toma son $U_{B}$ inversamente proporcionales al factor de tomas. Se aplica para tomas con factores de toma superiores al factor de toma de tensión máxima. (a)

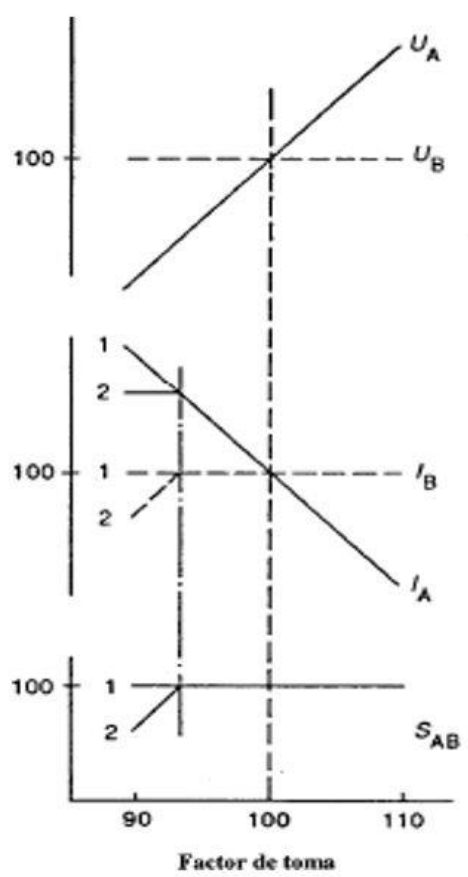

(b)

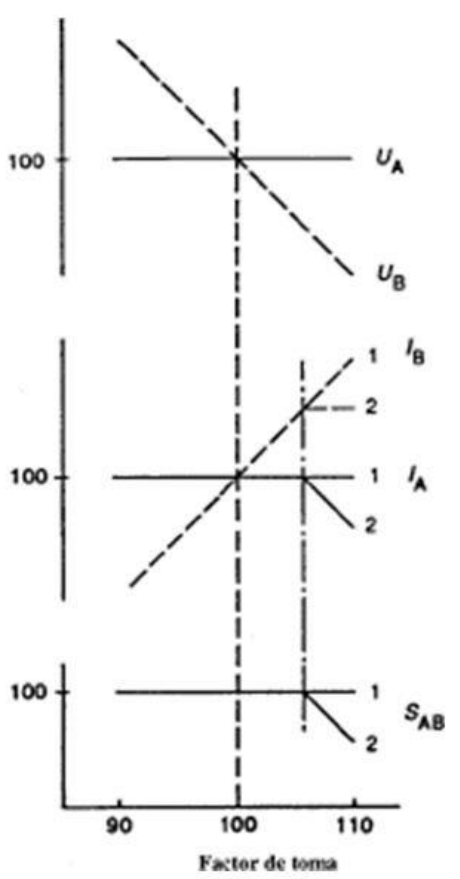

(c)

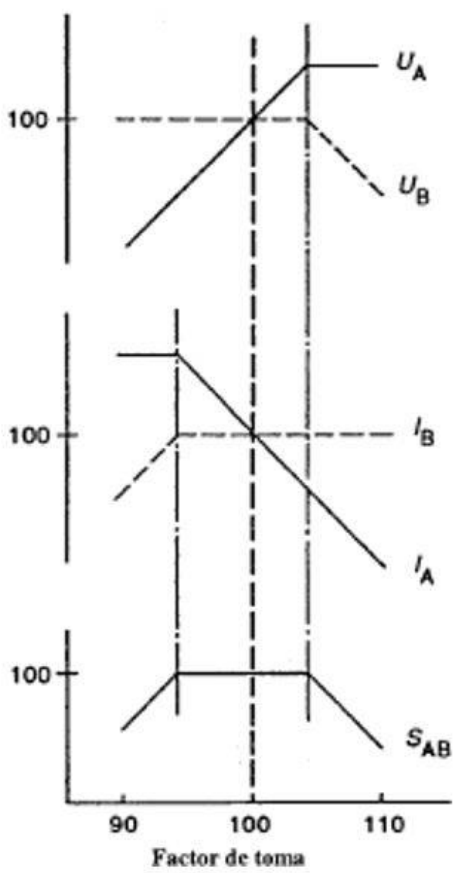

Fig. 2. (a) Regulación de tensión a flujo constante RFC. (b) Regulación de tensión a flujo variable RFV. (c) Regulación mixta RCb de acuerdo con el voltaje y factor de forma [4]

- Regulación de la tensión combinada o mixta RCb (figura 2.c): En muchas aplicaciones y sobre todo en transformadores con una gran extensión de tomas, se especifica una combinación utilizando ambos principios a distintas partes de la extensión, es decir, regulación de la tensión combinada. El punto de discontinuidad se denomina "toma de tensión máxima".

En la figura 2 además encontramos SAB corresponde a la potencia de toma. En la figura 2.a y 2.b las curvas 1 indica tomas de plena potencia a lo largo de la extensión de tomas, mientras que las curvas 2 tenemos Indica "toma de tensión máxima”, "toma de corriente máxima" y la extensión de las tomas de potencia reducida.

\subsection{CONSIDERACIONES DE DISEÑO}

Estas consideraciones son necesarias de comprender dado que en este caso el filtro está siendo incorporado en el transformador y utilizando las características de su reactancia, inductiva y capacitiva. En la extensión RFC el punto de discontinuidad aparece en la extensión de tomas aditivas, y constituye a la vez una toma de tensión máxima UA y una toma de corriente máxima IA, constante por encima del punto de discontinuidad. También aparece una toma de corriente máxima adicional y opcional. Todas las tomas estarán a plena potencia, salvo las indicadas a continuación:

- Para transformadores de arrollamientos separados de hasta 2500 kVA, con una extensión de tomas no superior a $\pm 5 \%$, la corriente de toma en el arrollamiento con tomas será igual a la corriente asignada en todas las tomas sustractivas. Esto significa que la toma principal es una "toma de corriente máxima". 
A. Yarin, et al.

- En transformadores con una extensión de tomas superior a $\pm 5 \%$, se pueden especificar restricciones sobre los valores de la tensión de toma o la corriente de toma, que de otra forma sobrepasarían considerablemente sus valores asignados. Cuando se especifiquen tales restricciones, las tomas relevantes serán "tomas de potencia reducida". Este apartado describe tales disposiciones.

Por otro lado, en relación con el factor de forma, cuando es distinto de la unidad, la corriente de toma, en tomas de plena potencia, puede sobrepasar la corriente asignada en uno de los arrollamientos. Como muestra la figura 2a, esto se aplica a las tomas sustractivas en el arrollamiento con tomas con una regulación RFC y, de acuerdo con la figura $2 b$, las tomas aditivas en el arrollamiento sin tomas, con una regulación RFV.

Para limitar el sobredimensionamiento del arrollamiento en cuestión, es posible especificar una toma de corriente máxima. Desde esta toma en adelante, los valores de la corriente de toma se fijan constantes. Esto significa que las tomas restantes hasta la toma extrema son tomas de potencia reducida.

(a)

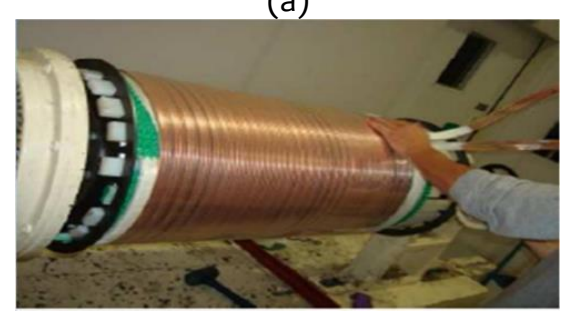

(b)

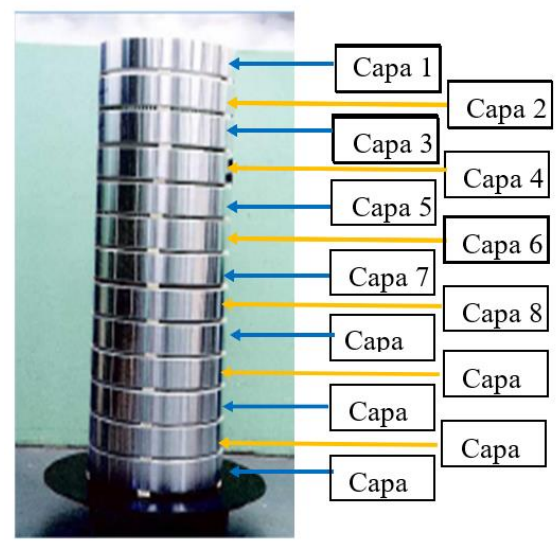

Fig. 3. Implementación de las bobinas de baja (a) y alta (b) tensión del transformador.

Salvo especificación contraria, con una regulación $\mathrm{RCb}$, la "toma de tensión máxima", en el punto de discontinuidad entre RFC y RFV, será igualmente una toma de "corriente máxima", es decir, la corriente del arrollamiento sin tomas permanece constante hasta la DOI: https://doi.org/ 10.21754/tecnia.v21i2.1031 toma aditiva extrema como en la figura 2c. Se debe considerar que habrá tantas capas como lo sea necesario para mantener la relación de transformación, evitando gradientes de potencial que dañen el aislante empleado.

En el caso de las bobinas de alta el daño es más crítico, dado que el aislamiento debe soportar tensiones mínimas del orden de $10 \mathrm{kV}$, al mismo tiempo que la rigidez eléctrica debe ser soportada por el aceite. De esta forma, cuanto mayor sea la capacitancia de las bobinas de alta mayor mayores serán las perdidas.

En estas condiciones aparece entonces la necesidad por desarrollar un elemento de protección que atenúe la presencia de estos armónicos y que posea un sistema de conexión automática efectiva del filtro, en el instante que se produzca este episodio. Para ello será necesario que exista una baja impedancia al armónico presente de modo que este sea conducido a tierra.

Para ello, de acuerdo a la figura 4, consideremos que las porciones de bobinados sobrantes U-1, V-2, W-3 luego de la elección del tap adecuado, pueden servir para formar el filtro pasivo, si se agrega un condensador sintonizado externo en la centralina entre los puntos 1 , 2 y 3 en el instante que se detecte el armónico presente estaremos satisfaciendo nuestros propósitos.

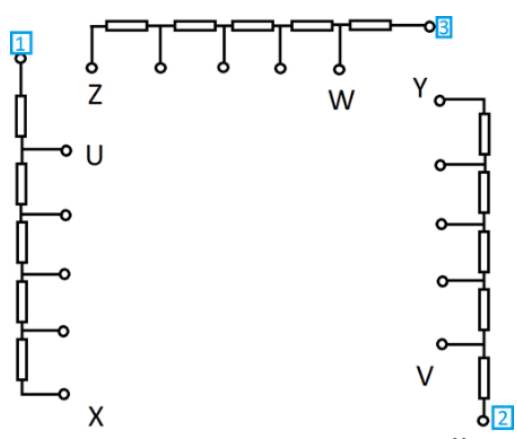

Fig. 4. Transformador con bobinas del filtro insertado.

\section{DISEÑO DEL FILTRO PASIVO 3.1 CARACTERIZACIÓN DE COMPONENTES $Y$ MATERIALES}

Los filtros pasivos comúnmente son unidades independientes al transformador, aun cuando trabajan con el fin de aislar los armónicos de las cargas sensibles no tienen mayor necesidad de proximidad 0 incorporación en la SS.EE. A continuación, se describen los componentes de un filtro:

- Condensador de media tensión: Es un arreglo de tres capacitores capaz de soportar la media tensión asociada a los armónicos. Estos deben ser de tecnología auto cicatrizante y auto-desconectable de tal modo que en presencia de una

Revista TECNIA Vol.31 Nº Enero-Junio 2020 
A. Yarin, et al.

descarga disruptiva interna abra el contacto hasta el cese de la sobrecarga. Este condensador irá alojado en una centralina próxima como se observa en la figura 05.

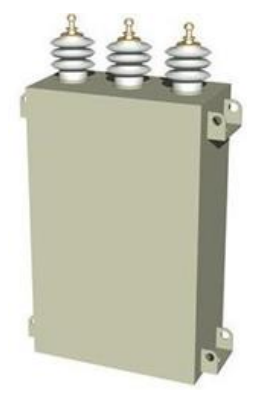

Fig. 5. Condensador de media tensión para incorporar al filtro.

- Centralina: Son tableros con un IP adecuado para alojar los capacitores de media tensión, con ventilación natural o forzada.

- Cable Seco: Este tipo de conductor es empleado en media tensión, y está provisto de una malla semiconductora que evita la presencia ionizante del campo eléctrico, tal como se muestra en la figura 06. Esto previene descargas producto de la perforación de la envolvente mencionada. Además, es preciso respetar un radio mínimo de curvatura en el desarrollo del cable, para evitar así el agrietamiento de la banda.

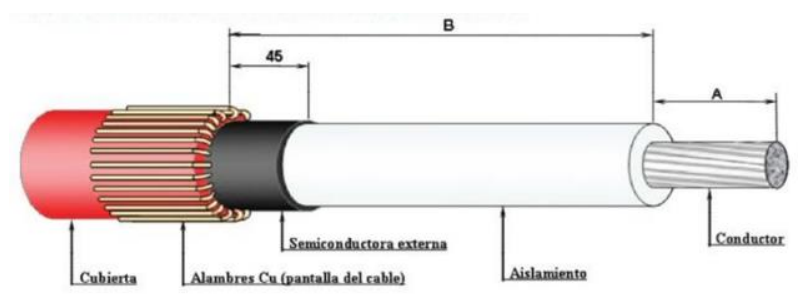

Fig. 6. Cable seco de media tensión.

- Terminales de Media Tensión: Dos líneas de 10kV próximas entre sí deben mantener una separación de tal modo que se reduzca la posibilidad de descargas por efecto corona, manifestado en los meses de invierno con mayor frecuencia. En este sentido cuando los cables llegan a aproximarse es sumamente útil que la llegada se realice con terminales de media tensión, los que gracias a su línea de fuga evitan las temidas descargas entre líneas energizadas.

\subsection{FACTORES FÍSICOS QUE AFECTAN EL DESEMPEÑO DEL FILTRO.}

Nos interesa particularmente el enfriamiento forzado, necesario debido a que la excesiva presencia de distorsión se manifiesta como ondas de calor que afectan directamente a las bobinas. Una ventilación o enfriamiento forzado es oportuna en estos casos, pues los condensadores pueden colapsar de otro modo. EI transformador tiene regímenes de trabajo variables, por lo tanto, hay que considerar que la temperatura va a saturar el núcleo, y a su vez ocasionar esfuerzos térmicos en los aislantes.

\subsection{DIMENSIONAMIENTO DE LA REACTANCIA CAPACITIVA CON MATLAB}

Dado que parte importante de la novedad del presente trabajo consiste en la incorporación de los parámetros capacitivos e inductivos del propio transformador en la sintonía del filtro pasivo es necesario validar dicho fenómeno en el proceso de filtrado [6]. Para esto nos apoyamos del software de Matlab donde implementamos un filtro con condiciones iniciales fijados mediante los criterios antes explicados. Finalmente, el filtro obtenido deberá tanto atenuar el THD como el factor de potencia. De este modo podemos obtener el siguiente resultado convergente:

Tabla I

Resultados código MATLAB

\begin{tabular}{|c|c|c|c|c|}
\hline $\begin{array}{c}\text { Li } \\
(\mathrm{mH})\end{array}$ & $\begin{array}{c}\text { Lp } \\
(\mathrm{mH})\end{array}$ & $\begin{array}{c}\text { Cf } \Delta \\
(\mu \mathrm{F})\end{array}$ & $\begin{array}{c}\text { Lo } \\
(\%)\end{array}$ & $\begin{array}{c}\text { THD de } \\
\text { línea }(\%)\end{array}$ \\
\hline 20.9659 & 9.0562 & $1.46 \mathrm{E}-06$ & 0.0254 & 4.4721 \\
\hline 20.9659 & 9.0562 & $9.71 \mathrm{E}-06$ & 0.0038 & 4.4559 \\
\hline 20.9659 & 9.0562 & $9.71 \mathrm{E}-05$ & $3.81 \mathrm{E}-04$ & 4.2907 \\
\hline 20.9659 & 9.0562 & $9.71 \mathrm{E}-04$ & $3.81 \mathrm{E}-05$ & 3.1039 \\
\hline
\end{tabular}

Nótese que el filtro también corrige el factor de potencia, que es una bondad adicional. Aquí se observa la inductancia total de sintonización Li y la efectiva del filtro Lp, además de la carga capacitiva del mismo $\mathbf{C f} \Delta$. Se observa que con cada cambio en las propiedades del filtro el THD se ve reducido lo mismo que Lo, que representa el porcentaje operativo comparativo del THD al aplicar el filtro respecto condiciones en las que no se utiliza.

\subsection{CARACTERÍSTICAS GENERALES DEL FILTRO}

- Tipo pasivo, es decir tiene inductancias ubicadas o formando parte del mismo bobinado del transformador.

- Factor de potencia de 0.99, que permite un ahorro de energía evidente.

- Inductancia de $20.1 \mathrm{mH}$

- Capacitancia de 0.97nF

Revista TECNIA Vol.31 Nº Enero-Junio 2020 


\section{MÉTODOS PARA LA OPTIMIZACIÓN EMPLEANDO ALGORITMO BACTERIAL FORAGIN OPTIMIZATION ALGORITHM}

El Algoritmo de Optimización de Forraje de Bacterias (BFOA), propuesto por Passino [2], es un nuevo miembro de la familia de algoritmos de optimización inspirados en la naturaleza. Durante las últimas cinco décadas, la optimización por algoritmos, como Algoritmos Genéticos (GA), Programación Evolutiva (EP), Estrategias Evolutivas (ES), que se inspiran en la evolución y la genética natural, han ido dominando el reino de los algoritmos de optimización. Recientemente algoritmos inspirados en enjambres naturales como optimización de enjambre de partículas (PSO), Ant Colony Optimization (ACO) y otros, han encontrado su camino en este dominio y demostrado su eficacia [2].

Siguiendo la misma tendencia de algoritmos basados en enjambres. El BFOA en aplicación de la estrategia grupal de alimentación de un enjambre de bacterias escherichia coli, donde las bacterias realizan una optimización de búsqueda en base a funciones multi-óptimas, siendo esta la idea clave del nuevo algoritmo.

La búsqueda de nutrientes que realizan las bacterias para maximizar la energía obtenida por unidad de tiempo, es motivo de un despliegue incesante por alcanzar la sobrevivencia de la colonia bacterial. La bacteria individual también se comunica con las demás enviando señales, en la cual una bacteria toma decisiones de alimentación después de considerar dos factores:

- La calidad y cantidad de los nutrientes ubicados que van a definir los pasos chemotácticos de avance.

- La amenaza que representa la región de búsqueda explotada, mediante la cual se efectúa la eliminación de las bacterias que tomaron la decisión de avance a expensas de su supervivencia.

Para modelar matemáticamente este proceso, es necesario visualizar el proceso de optimización como una simulación del movimiento descrito por una célula de escherichia coli. Se toman en cuenta los siguientes pasos para lograrlo [5]:

A. Biológicamente una bacteria E. coli puede desplazarse en dos trayectorias diferentes. Puede nadar en una sola trayectoria en un lapso de tiempo o puede cambiar de entorno rápidamente. Ambos modos de funcionamiento pueden modelarse con $\Phi^{i}(j, k, l)$ que representa a la i-ésima bacteria en el j-avo paso chemotáctico, la etapa de eliminación-dispersión reproductiva y l-ésimo orden k.

B. C(i) es el tamaño de la etapa de tomada en una dirección definida probabilísticamente. Luego, en modelo computacional la chemotaxis puede ser representado por

$\Phi^{i}(j+1, k, l)=\Phi^{i}(j, k, l)+C(\mathrm{i}) * \frac{\Delta(i)}{\sqrt{\Delta^{T}(i) \Delta(i)}}$

C. Donde $\Delta$ indica un vector en dirección aleatoria cuyos valores están en $[-1,1]$.

D. El modelamiento también debe contemplar la comunicación que tiene las baterías para la búsqueda de nutrientes y de buenas condiciones de reproducción. Por ende, este intercambio de señales puede representarse por la función (2).

$$
\begin{aligned}
& J_{c c}(\theta, P(j, k, l))=\sum_{i=1}^{p} J_{c c}\left(\theta, \theta^{i}(j, k, l)\right) \\
& J_{c c}(\theta, P(j, k, l))=\sum_{i=1}^{S}\left|-d_{\text {attractant }} \exp \left(-I I I_{\text {attractant }} \sum_{m=1}^{p}\left(\theta_{m}-\theta_{m}^{i}\right)^{2}\right]+\sum_{i=1}^{S}\right| h_{\text {repellant }} \exp \left(-I I I_{\text {repellant }} \sum_{m=1}^{p}\left(\theta_{m}-\theta_{m}^{i}\right)^{2} \mid\right.
\end{aligned}
$$

Donde:

- $J_{c c}(\theta, P(j, k, l))$ es el valor de la función objetivo que se añade al objetivo de función real (que se reduce al mínimo) para presentar un tiempo que varía de la función objetivo.

- S es el número total de bacterias
- $p$ es el número de variables que deberá ser optimizado, y que están presentes para cada bacteria

- $T_{p}=1,2, \ldots, q=q_{n}$ es un punto en el dominio de búsqueda $\mathrm{p}$ dimensional. 
A. Yarin, et al.

E. Reproducción: Las bacterias menos saludables eventualmente mueren, mientras que cada una de las bacterias saludables (aquellos con rendimiento de menor valor de la función objetivo) asexualmente dividido en dos bacterias quedan reproducidos.

F. Finalmente, mediante código Matlab, se busca minimizar la función de Rosembroch, que luego va a tomar la forma de la ecuación (2). Aquí J es la función costo que se desea minimiza, se parte de 20 bacterias, considerando una probabilidad de eliminación dispersión del $90 \%$, es decir que 18 bacterias se pueden sacrificar a fin de lograr el óptimo.

\section{SOFTWARE NEPLAN}

Uno de los grandes problemas a los que se enfrenta la modelación de eventos energéticos de potencia eléctrica usando el método circuital de flujo de carga, es la veracidad de los resultados. Si bien es cierto que este método es exacto desde sus fundamentos, tenemos que añadir un factor de error dados los fenómenos físicos que no contempla, como el efecto térmico, y los problemas para generar diseños fiables.

Para implementar la simulación en NEPLAN se utilizaron módulos de Distribución como se muestra en la figura 07. Estos módulos empleados en el análisis pueden asociarse con tablas dinámicas de Excel para incorporar data de los fenómenos que no contempla por cuenta propia el software.

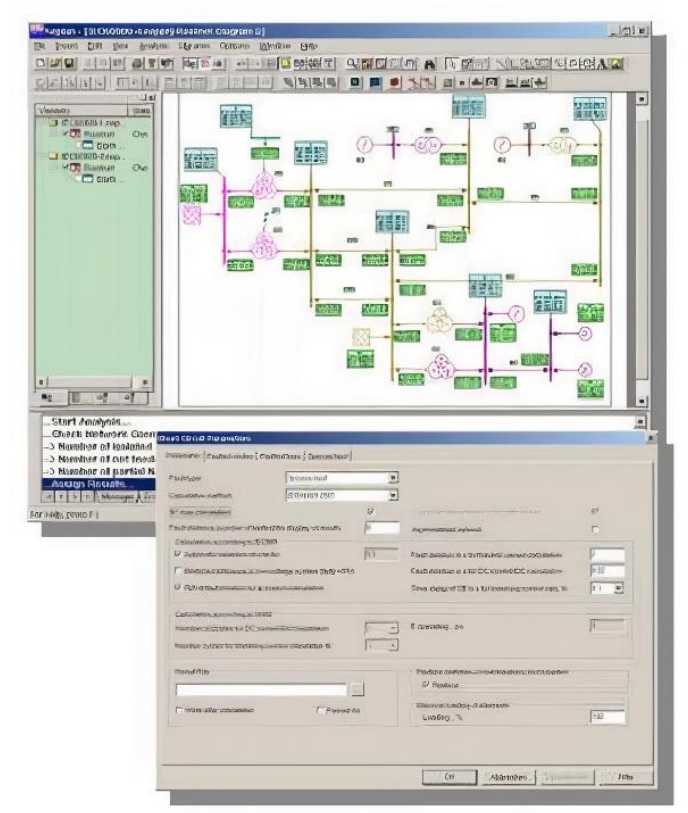

Figura 07. Trabajo de simulación en NEPLAN

\section{CONDICIONES EXPERIMENTALES.}

El desempeño del filtro tiene dificultades desde que la presencia misma del armónico produce calor y con ello el deterioro temprano de los componentes. Este calentamiento nos lleva a sobredimensionar el núcleo y los conductores que con reducida sección soportarían. La clase de aislamiento $\mathrm{F}$ permite un calentamiento de $155^{\circ} \mathrm{C}$ y la clase $\mathrm{H}$ de $180^{\circ} \mathrm{C}$. Ello nos obliga a diseñar un enfriamiento oportuno que mantenga la temperatura en el interior de la máquina debajo de estos límites.

Para la experiencia realizada con un transformador de 80okVA se tienen las siguientes condiciones similares a la de una SS.EE.:

- Distorsión de corriente del 28\% de THDi

- Temperaturas del orden de $66^{\circ} \mathrm{C}$

- Potencia de $60 \%$ de plena carga

Bajo estas condiciones se estimó que en el interior la temperatura debe bordear los $90^{\circ} \mathrm{C}$.

\section{RESULTADOS}

- Se ha logrado atenuar lo distorsión armónica hasta lograr un THD de $3.13 \%$ de la onda fundamental (Ver tabla 4.1). Ello representa gran confiabilidad si consideramos que la norma IEEE519-92 considera un máximo permisible de $10 \%$. Resultados similares se obtuvieron mediante un filtro activo SAPF controlado mediante algoritmo auto-tunning PSO-PI en [17], e incluso mejores que en [18], donde se aplica el algoritmo de forraje bacterial a un filtro SAPF directamente a su respuesta mediante PSO-PI.

- Se logró insertar un filtro aprovechando las bobinas del propio transformador, que quedan sin uso luego de seleccionar los taps óptimos. Y con ello hacer más robusta la SS.EE frente a la amenaza de distorsión no senoidal.

- La ubicación de estos taps de derivación fue seleccionada mediante el algoritmo de forraje bacterial gracias a su capacidad de considerar varias posiciones óptimas simultáneamente mediante la función costo asociada al filtro.

- El calentamiento, que es factor ineludible, debe controlarse mediante ventilación forzada, de acuerdo a la necesidad.

- El costo se ve optimizado desde que el transformador puede soportar episodios críticos de amenazas de armónicos, evitando su colapso y con ello la confiabilidad de la SS.EE.

Revista TECNIA Vol.31 Nº1 Enero-Junio 2020 
A. Yarin, et al.

La efectividad del filtro pasivo propuesto es equivalente al de filtros activos de alta eficiencia que a la vez han sido optimizados mediante algoritmos de control PI de selftunning mediante PSO y también mediante aplicación directa del de forraje de partículas. La principal diferencia del presente trabajo radica tanto en la optimización de un filtro pasivo como su

\section{REFERENCIAS}

[1]L. Arrillaga y L. Eguíluz, Armónicos En Sistemas De Potencia. España: Universidad de Cantabria ,1994.

[2]J. Brown, Clever Algorithms: Nature-Inspired Programming Recipes. Lulu, 2012.

[3] A. García y X. Alabern, Instalaciones Eléctricas. Barcelona: Marcombo Ediciones Técnicas, 2002.

[4]E. Kimbark, Direct Current Transmission, USA: Wiley, 1981

[5]M. Mohammadi, "Direct Bacterial foraging optimization and adaptive version for economically optimum sitting sizing and harmonic", Applied Soft Computing, vol. 29, pp. 345-356, abr. 2015.

[6]l. Pérez, "Cálculo de parámetros de filtros pasivos de armónicos", Energética, vol. 33, no 2, pp. 133-142, ago. 2012

[7]J. Váquez, J. Flores, P. Salmerón, S. Litrán, “Diseño de filtros pasivos, activos e híbridos para la compensación armónica de cargas trifásicas no lineales", Asociación Española para el Desarrollo de la Ingeniería Eléctrica, 2004

[8]H. Zubi, "Lowpass broadband harmonic filter design". Tesis Magister, Ankara, Universidad Técnica De Oriente Medio, Turquía, 2005.

[9]P. Morshuis, P. Morshuis, J. Smit, A. Janssen y E. Gulski, "A statistical approach to processing power transformer failure data", en International Conference on Electricity Distribution, Vienna, 207, pp1-4.

[10]J. Jagers y S. Tenbohlen, "Evaluation of transformer reliability data based on national and utility statistics", Eskom Research and Innovation y Universität Stuttgart, 2009 incorporación en las propias bobinas del transformador mediante los taps indicados por el algoritmo de optimización de forraje bacterial. De esta manera, se presenta una nueva oportunidad para el estudio de filtros pasivos y una revisión de sus propiedades mediante este método de optimización y en condiciones de operación diferentes.

[11]C. Orellan, "Análisis de distorsión armónica en redes de distribución eléctrica utilizando Matlab", Trabajo de Gradiación, Ciudad de Guatemala, Universidad de San Ancarlos de Guatemala, Guatemala, 2011.

[12]IEEE, "IEEE Recommended Practice and Requirements for Harmonic Control in Electric Power Systems," in IEEE Std 519-2014 (Revision of IEEE Std 519-1992), vol., no., pp.1-29, 11 June 2014, doi: 10.1109/IEEESTD.2014.6826459

[13]F. Vahidi y S. Tenbohlen, "Statistical failure analysis of European substation transformers". En Conferencia: 6. ETG-Fachtagung Diagnostik elektrischer Betriebsmittel 2014, Baden-Württemberg. [14]J. Henning, L. Nordström y P. Hilber, "Individual failure rates for transformers within a population based on diagnostic measures", Electric Power Systems Research, vol. 141, pp. 354-363, dic. 2016

[15]G. Massalin, "Analysis and evaluation of harmonic distortion in industrial networks caused by HVAC air-conditioning systems", Tesis de Master, Padova, Universidad de Padua, Italia, 2020

[16]J. Arcila, "Armónicos en sistemas eléctricos", IEB SA, pp. 1-25 2020 [17]E. Mawhi, H. Bin y Herwan Mohd, "Performance analysis of active power filter for harmonic compensation using PI-PSO", ARPN Journal of Engineering and Applied Sciences, vol. 10, no 21, pp. 9885-9891, nov. 2015.

[18] G. El-Saady, E. A. Ebrahim, H. I. Abdul-Ghaffar, Y. S. Mohamed y A. El-Sayed, "Particle-Swarm Optimization Control of Active-Power Filter for Harmonic Mitigation of Hybrid Electric-Unbalanced TractionSystem," 2018 IEEE Congress on Evolutionary Computation (CEC), 2018, pp. 1-8, doi: 10.1109/CEC.2018.8477653.

[19]Norma Europea UNE-EN60076-1. “Comité Técnico AEN/CTN 207. Transporte y distribución de energía eléctrica”, 2011. 
APENDICE 1:

Medición de Capacitancia con CPC 100Omicrom

\section{Capacitancia de los devanados}

\begin{tabular}{|c|c|c|c|}
\hline $\begin{array}{c}\text { Modo de } \\
\text { prueba }\end{array}$ & $\begin{array}{c}\text { Tensión de } \\
\text { Prueba } \\
(\mathbf{k V})\end{array}$ & Capacitancia (pF) & EVALUACION \\
\hline $\mathrm{CH}+\mathrm{CHL}$ & 10 & 5597.35 & $\left({ }^{*}\right)$ \\
\hline $\mathrm{CH}$ & 10 & 2985.84 & $\left({ }^{*}\right)$ \\
\hline $\mathrm{CHL}(\mathrm{UST})$ & 10 & 2611.64 & $\left(^{*}\right)$ \\
\hline $\mathrm{CL}+\mathrm{CLT}$ & 10 & 5268.48 & $\left(^{*}\right)$ \\
\hline $\mathrm{CL}$ & 10 & 1612.07 & $\left({ }^{*}\right)$ \\
\hline $\mathrm{CLT}$ & 10 & 3655.56 & $\left(^{*}\right)$ \\
\hline $\mathrm{CT}+\mathrm{CHT}$ & 10 & 3078.51 & $\left(^{*}\right)$ \\
\hline $\mathrm{CT}$ & 10 & 2914.43 & $\left({ }^{*}\right)$ \\
\hline $\mathrm{CHT}$ & 10 & 164.114 & $\left({ }^{*}\right)$ \\
\hline
\end{tabular}

Fuente: Cortesía de T\&D Electric S.A 
APENDICE 2:

Características del equipo CPC 1000microm

Equipo de Prueba Primarias.

\begin{tabular}{|l|l|l|l|}
\hline Marca & OMICRON & Modelo & CPC 100 \\
\hline$N^{\circ}$ Serie & MF117T & $\begin{array}{l}\text { Año de } \\
\text { Fabricación }\end{array}$ & 2011 \\
\hline
\end{tabular}

Equipo de Prueba Primarias.

\begin{tabular}{|l|l|l|l|}
\hline Marca & OMICRON & Modelo & CP TD1 \\
\hline$N^{\circ}$ Serie & KK427S & $\begin{array}{l}\text { Año de } \\
\text { Fabricación }\end{array}$ & 2011 \\
\hline
\end{tabular}

